\title{
Human Perception of Laid-Out Graphs
}

\author{
Edmund Dengler and William Cowan \\ Department of Computer Science, \\ University of Waterloo, \\ Waterloo, Ontario, N2L 3G1
}

\begin{abstract}
Combinatorial graphs are increasingly used for information presentation. They provide high information density and intuitive display of multiple relationships, while offering low cost because they can be created algorithmically. Essential to algorithmic graph layout is a set of rules that encode layout objectives. How these rules are related to inferences drawn from the graph by human observers is a largely unexplored issue. Thus, success or failure by algorithmic standards is only uncertainly related to perceptual effectiveness of the resulting layout. Human experimentation is the only way to correct this deficiency.

This poster describes empirical research conducted in 1994. Forty-six respondents, separated into naive and computer-aware groups, freely viewed a collection of graph layouts, providing semantic conclusions they reached on the basis of the layout, in the absence of any semantic attribution to nodes in themselves. We were interested in two questions. First, are semantic attributions consistent or random? If the former semantic objectives must be considered when creating layout rules or objective functions for automated graph layout. Second, if consistent semantic attributions exist, what are they? The remaining paragraphs of this abstract describe our results and conclusions.

Most importantly, all our observers agreed strongly as to the semantic content of specific graph layouts. There was no difference in interpretation between the group consisting of experienced programmers, and the group who had little exposure to computers. We were interested in possible differences because combinatorial graphs are extremely common in computer science curriculum material, and it's possible that a group of programmers might agree because they had been exposed to a common set of layout conventions. The agreement between our two groups demonstrates that semantic conventions extend widely.

The most important semantic attribution is that observers view graph layouts hierarchically. Graphs are separated into interconnected subgraphs. Both the relative layout of the subgraphs and the layout within each subgraph are interpreted semantically. Thus, it is possible to encapsulate layout semantics in terms of relationships in graph layouts that have relatively few nodes. A few rules that we observed to be used by more than 90

1. Nodes positioned symmetrically, whether in a circle, on a line or on a grid, are interpreted as having properties in common, or as being equal in status.
\end{abstract}


2. Nodes positioned centrally, and nodes positioned nearer the top of the layout are interpreted as having special properties, or as being higher in status.

3. When nodes are arranged linearly, it is assumed that the sequence is significant, with the sequence going from left-to-right or from top-to-bottom.

4. Drawn edges are used in determining interpretations of patterns. For example, the left figure is interpreted as a special node that is superior to two similar nodes, while the right figure is interpreted as three similar nodes.
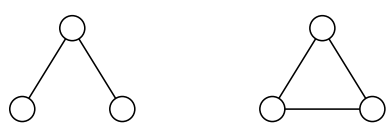

5. The absence of any of the above features is interpreted semantically as the absence of the interpreted quality. For example, the figure below shows three layouts of a K8 graph with one edge missing. The right layout maximizes symmetry and minimizes edge crossings (traditional aesthetic layout principles), however, it is difficult to discern immediately that an edge is missing. The middle layout breaks symmetry to allow quick identification of an anomalous state. The right layout breaks the symmetry of the edges in a manner that allows finding the missing edge easily, although more edge crossings are introduced.
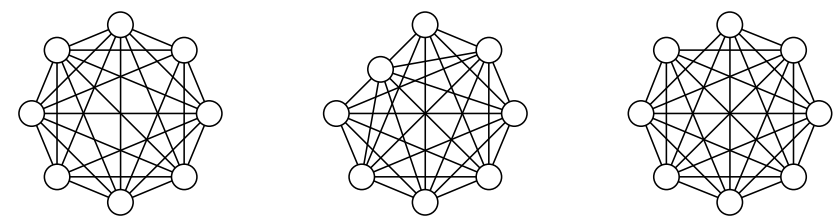

Another example shows how different layouts affect semantic interpretation of the same combinatorial graph. The left layout below is interpreted as a hierarchy of two elements controlling three others, whereas the right layout is interpreted as five equivalent elements. The absence and presence of different features creates different semantic meanings.
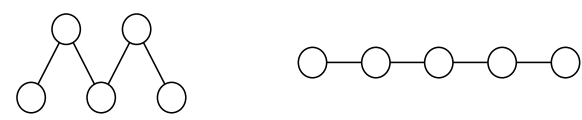

We don't claim that this list is comprehensive. The relationship of features like the ones we discovered to principles of perceptual organization, like those discovered by Gestalt psychologists, is clearly required to put our results onto a more solid quantitative footing. What is, however, abundantly clear from our 
experiments is that perceptually effective graph layout requires layout rules and objective functions that are far more complex than those in current use. The algorithmic consequences of the necessarily broadening of layout objectives remains to be explored.

This project was the thesis research of the first author. The thesis, which provides a comprehensive description of the research, is available by e-mailing him at

EADengler@cgl. UWaterloo.ca. 